\title{
Antibiotic regimens for neonatal sepsis - a protocol for a systematic review with meta- analysis
}

Steven Kwasi Korang ${ }^{1 *}$ (D), Sanam Safi ${ }^{1}$, Christian Gluud ${ }^{1}$, Ulrik Lausten-Thomsen ${ }^{2}$ and Janus C. Jakobsen ${ }^{1,3,4}$

\begin{abstract}
Background: Sepsis is a major cause of morbidity and mortality among neonates and infants. Antibiotics are a central part of the first line treatment for sepsis in neonatal intensive care units worldwide. However, the evidence on the clinical effects of the commonly used antibiotic regimens for sepsis in neonates remains scarce. This systematic review aims to assess the efficacy and harms of antibiotic regimens for neonatal sepsis.

Methods: Electronic searches will be conducted in MEDLINE, Embase, The Cochrane Library, CINAHL, ZETOC and clinical trial registries (clinicaltrials.gov and ISRCTN). We will include randomised controlled trials of different antibiotic regimens for sepsis of neonates and infants. Eligible interventions will be any antibiotic regimen. Two reviewers will independently screen, select, and extract data. The methodological quality of individual studies will be appraised following Cochrane methodology. Primary outcomes will be 'all-cause mortality' and 'serious adverse events'. Secondary outcomes will be 'need for respiratory support', 'need for circulatory support', 'neurodevelopmental impairment', ototoxicity, nephrotoxicity and necrotizing enterocolitis. We plan to perform a meta-analysis with trial sequential analysis.
\end{abstract}

Discussion: This is the study protocol for a systematic review on the effects of different antibiotic regimens for neonatal sepsis. The results of this systematic review intent to adequately inform stakeholders or health care professionals in the field of neonatal sepsis, and to aid appropriate development of treatment guidelines.

Systematic review registration: PROSPERO reference number: CRD42019134300.

Keywords: Sepsis, Neonates, Infants, Septic shock, Antibiotics, Systematic review

\section{Background}

Description of the condition

\section{Definition}

Sepsis occurring before 28 days after birth is termed neonatal sepsis $[1,2]$. There is currently no international consensus on the definition of neonatal sepsis [3, 4]. Most neonatal sepsis criteria used in clinical trials are based on different combinations of clinical and laboratory parameters [4-6].

Due to the lack of consensus on the definition of neonatal sepsis, it is difficult to estimate the exact incidence of neonatal sepsis [1]; however, the incidence is

\footnotetext{
* Correspondence: steven.korang@ctu.dk

${ }^{1}$ Copenhagen Trial Unit, Centre for Clinical Intervention Research,

Department 7812, Copenhagen University Hospital, Rigshospitalet,

Copenhagen, Denmark

Full list of author information is available at the end of the article
}

estimated to be between 1 and 12 per 1000 live births in high-income countries [1]. The incidence in low- and middle-income countries is higher, and in Asia, the incidences have been estimated to be up to 38 per 1000 live births [7-12].

Neonatal sepsis is a major cause of morbidity and mortality. It is the third leading cause of neonatal mortality and constitutes $13 \%$ of overall, global neonatal mortality $[13,14]$. In the high-income countries, neonatal sepsis has a mortality ranging from 5 to $20 \%$ and causes major disability (or death) in up to $40 \%$ of all cases despite initiation of conventional treatment [1]. Mortality rates up to $70 \%$ have been observed in some low- and middle-income countries $[1,4,15,16]$.

In survivors, sepsis is associated with serious longterm morbidity such as cerebral palsy, cognitive and 
psychomotor delay, auditory and visual impairment, and bronchopulmonary dysplasia [1, 17-19]. Most of these associations are based on observational cohort studies and therefore does not distinguish between causality and association. It remains uncertain whether it is possible to prevent these subsequent sequela by treating neonatal sepsis with appropriate empirical antibiotic regimens [1].

Depending on the time of onset, neonatal sepsis may be divided into early onset sepsis and late onset sepsis. The most commonly accepted distinction between these two subgroups is before and after $72 \mathrm{~h}$ (but other definitions, e.g. $48 \mathrm{~h}$ and 7 days exist) $[1,2,20-26]$. This distinction is based on the assumed different aetiologies and pathophysiology of pathogens typically seen before and after $72 \mathrm{~h}[2,22,27]$.

The infection in early onset sepsis is usually acquired vertically from a colonised mother, while the infection in late onset sepsis is usually acquired horizontally, e.g. from the community or a nosocomial (hospital-acquired) infection [16, 22, 26, 28, 29]. However, these theoretical differences might not warrant a need for different antibiotic regimes for early and late onset neonatal sepsis, especially if broad-spectrum antibiotics are used. Accordingly, several trials have included both types of neonates without distinguishing between early and late onset sepsis. As the clinical manifestations also can be non-specific, it can be hard to clinically distinguish between sepsis and deep-seated infections such as meningitis, osteomyelitis and necrotizing enterocolitis $[2,26]$.

The pathogens causing neonatal sepsis include grampositive and gram-negative bacteria [30]. The mortality and the distribution pattern of pathogens causing sepsis in neonates differs between low- and middle-income countries and high-income countries. Important pathogen variations can sometimes even be seen between individual neonatal intensive care units (NICUs) within a given country. Furthermore, the predominant organisms responsible for neonatal sepsis within regions have also changed with time [31, 32].

Neonates are theoretically immunocompromised as several components of the immune system are not fully developed at birth [2,33]. This is especially true for preterm newborns, as they are additionally immunocompromised due to an even more immature immune system [34-38]. Prematurity and low birth weight are therefore major risk factors and accordingly, a multicentre observational study showed that neonatal sepsis were most common in premature (82\%) and low birth weight neonates (81\%) [39].

Several other risk factors have been shown to be associated with an increased risk of developing neonatal sepsis [21].

For early onset neonatal sepsis, the risk factors are multiple gestation, maternal intrapartum fever, maternal urinary tract infection or chorioamnionitis, prolonged labour, preterm rupture of the membrane (PROM), prolonged PROM $>18 \mathrm{~h}$, and meconium aspiration syndrome $[27,40]$.

Late onset sepsis also has several risk factors such as mechanical ventilation, intravascular catheterisation, failure of early enteral feeding with breast milk, a prolonged duration of parenteral nutrition, surgery, underlying respiratory and cardiovascular diseases, and hospitalisation $[30,41-44]$.

\section{Description of the intervention}

Treatment of neonatal sepsis is aimed at treating the underlying infectious cause of sepsis [45, 46], and correcting the associated organic dysfunction through, e.g. respiratory support, circulatory support and correction of metabolic, temperature and glucose derangements $[47,48]$. This review assesses the first part, which is to treat the underlying infectious cause of sepsis.

Preliminary results have shown that early initiation of antibiotic therapy in neonates with suspected sepsis seems to reduce both mortality and morbidity [1]. According to guidelines, the treatment should be given as soon as possible and always within $1 \mathrm{~h}$ of the decision to treat [49]. The antibiotic therapy is empirical and based on several factors such as age at onset, likely pathogens, and antibiotic susceptibility patterns [21, 31, 50,51].

Among the most common types of antibiotics used for treatment for neonatal sepsis are beta-lactams (e.g. penicillins, cephalosporins, monobactams and carbapenems), aminoglycosides (e.g. gentamycin) and glycopeptides (e.g. vancomycin and teicoplanin) $[52,53]$.

The most commonly recommended and used first-line treatment for both early and late onset neonatal sepsis is a beta-lactam antibiotic (most commonly ampicillin, flucloxacillin and penicillin) combined with an aminoglycoside (most commonly gentamicin) [21, 31, 48, 51, 54-57]. However, there has been an increased use of alternative protocols using a cephalosporin (most commonly cefotaxime) or a glycopeptide (most commonly vancomycin) as a first line option to treat especially late onset sepsis [58-60], due to increased resistance among the most common pathogen such as coagulase-negative staphylococci $[31,50]$. Ampicillin combined with a third-generation cephalosporin agent (most commonly cefotaxime) is also used as an alternative for early onset sepsis $[39,54,58-60]$. Other regimens such as cephalosporins (as monotherapy) are also used [49]. Guidelines may differ due to local antibiotic resistance of the most common pathogens or whether the empirical regimen is supposed to cover the common but low virulence coagulase-negative staphylococci (for late onset sepsis) [61, 62]. Vancomycin is often considered if staphylococcal infection is suspected [63]. 
The duration of the antibiotic treatment is adjusted according to the type of pathogen, treatment response, and the possibility of the antibiotic to penetrate to the site of infection in case of, e.g. meningitis, encephalitis, osteomyelitis or endocarditis. A prospective observational study showed that $63 \%$ of neonates started in antibiotic therapy were discontinued within $48 \mathrm{~h}$ when cultured [58]. When and if a pathogen is identified by cultures, the antibiotic therapy might be changed according to the antibiotic susceptibility of the pathogen. However, causative bacteria are identified only in about one-third of the patients with presumed sepsis [33, 64, 65]. One study found that the empirical antibiotic regimen was changed in $44 \%$ of the cases when the pathogen and susceptibility was identified; the most frequently added antibiotics were vancomycin, cefotaxime and penicillin [39]. It is recommended to stop the antibiotic treatment when no signs and symptoms of infection is observed, and no pathogen is identified $[2,55]$.

\section{Antibiotic susceptibility}

Antibiotic resistance is a global and growing problem which increases the morbidity, mortality and costs associated with infections [57, 66-68]. The bacterial resistance to antibiotics results mainly from the selective pressure exerted by the use and overuse of antibiotics [67, 69-72]. Studies, comparing antibiotic susceptibility over time in the same unit, show increased resistance to the most used antibiotics [57].

The pathogens causing neonatal infections and their antibiotic susceptibility patterns change over time and may differ among countries [57, 73-77]. When comparing the epidemiology of neonatal sepsis in the low- and middle-income countries with the high-income countries, some important differences emerge in the pattern of etiological pathogens and their antibiotic resistance [11, 78-80].

In high-income countries, data from the UK showed that $95 \%$ of the identified pathogens were susceptible to the most commonly used empirical antibiotic regimens of penicillin and gentamicin [54]. In low- and middleincome countries, estimations suggest that up to $70 \%$ of pathogens isolated from neonatal sepsis may not be covered by the recommended empirical antibiotic regimen of ampicillin and gentamicin [81]. Some studies in the low- and middle-income countries have shown almost universal antibiotic resistance (92-100\% resistant) among the most common pathogens (gram-negative rods) to first-line (often ampicillin and gentamicin) and second-line antibiotics such as the third-generation cephalosporins [15, 48, 81].

In addition, some low- and middle-income countries face widespread dissemination of resistant bacterial strains, including extended-spectrum-lactamase-producing bacteria and methicillin-resistant Staphylococcus aureus (MRSA) [81-84].

\section{Adverse effects}

The use of anti-bacterial agents is potentially associated with adverse effects, but the published data in neonates are scarce and occasionally contradictory.

The use of ampicillin has in some studies been associated with adverse effects such as rashes, diarrhoea, nausea and nephrotoxicity $[52,53,85]$. Contrary to these findings, a recent systematic review of randomised clinical trials showed that ampicillin only increased the incidence of candidiasis with no significant increase in the abovementioned adverse effects [86]. Nephrotoxicity has been estimated to be rare (0.03\%) [85].

Aminoglycosides have been shown to be toxic (nephrotoxic and ototoxic) in adults, whereas its toxicity in neonates remains unclear [87-95].

The most common adverse effects caused by glycopeptides, i.e. vancomycin is fever and phlebitis and in rare cases nephrotoxicity and ototoxicity [96]. There are limited data suggesting a direct causal relationship between toxicity and specific serum vancomycin concentrations [96]. However, in addition to the development of resistance towards vancomycin some observational studies also suggest a three- to fourfold increase in nephrotoxicity when aminoglycosides are combined with vancomycin [96-100].

Cefotaxime is associated with increased risk of death and invasive candidiasis in non-randomised studies [59, 63, 101].

In addition to the specific adverse effects of each antibiotic, extended use of any antibiotics is also associated with higher risk of neonatal candidemia [102, 103].

\section{How the intervention might work}

Antibiotics are antimicrobial drugs that treat and prevent bacterial infections by either killing (bactericidal) or inhibiting the growth of the bacteria (bacteriostatic) [104]. They can be classified based on (1) their mechanism of action (bactericidal or bacteriostatic); (2) bacterial spectrum (broad or narrow); and (3) chemical structure (e.g. penicillins, macrolides, quinolones, tetracyclines or aminoglycosides) [105].

A combination of different antibiotics might have several advantages. Firstly, it is thought to provide an enhanced effect beyond the additive effects of the individual therapies [106]. Secondly, it can be used to broaden the spectrum of antibiotic coverage when used empirically to increase the chance of covering the presumed causative bacteria. Thirdly, a combination therapy is thought to suppress the development of subpopulations of microorganisms resistant to antibiotics [106-108]. 
However, it is theoretically possible that the optimal empirical antibiotic treatment should not be chosen solely based on the presumed pathogen and cultures. Antibiotics might have different effects in the human body compared with the pattern they show from in vitro (cultures).

\section{Why it is important to do this review}

Despite the high burden of neonatal sepsis, high-quality evidence in diagnosis and treatment is scarce [26]. Yet, in adults, appropriate empirical antibiotic treatment has been shown to halve the fatality associated with sepsis compared with inappropriate empirical antibiotic treatment [109-111].

Due to the diagnostic challenges of sepsis and the relative immunosuppression of the newborn, many neonates receive antibiotics for suspected sepsis. In fact, antibiotics have become the most commonly used pharmacological therapeutic in neonatal intensive care units [112]. Studies suggest that up to $95 \%$ of newborns treated with antibiotics for suspected sepsis prove to have no evidence of infection [58, 113, 114]. This presumed overuse of antibiotics seems to contribute to the development and spread of resistant pathogens in the neonatal intensive care units and seems to be associated with adverse events (e.g. invasive candidiasis and increased antimicrobial resistance) [67, 101, 112, 115-117]. Adverse effects of antibiotic exposure in infants is believed to be minimised through the appropriate antibiotic choice and duration of treatment [24].

To create the most appropriate antibiotic policies for neonatal sepsis, there is a need to base these policies on an updated systematic review with meta-analysis.

The latest two Cochrane reviews are from 2004 and 2005 and does not include trials not distinguishing between early and late onset sepsis [118, 119]. Both reviews concluded that there is inadequate evidence from randomised trials in favour of any particular antibiotic regimen for the treatment of suspected early and late onset neonatal sepsis, respectively [118, 119]. No other systematic review has been conducted to assess the effects of different antibiotic regimens for suspected neonatal sepsis regardless of onset.

There is therefore a need for a systematic review to assess the effects of different antibiotic regimens for neonatal sepsis taking into account both risks of systematic errors and random errors [120].

\section{Objectives}

The objective of this study is to compare the beneficial and harmful effects of different antibiotic regimens for neonatal sepsis.

\section{Methods}

The present protocol is being reported in accordance with the reporting guidance the preferred reporting items for systematic reviews and meta-analyses protocols (PRISMAP) statement [121]. This protocol has been registered within the PROSPERO database (CRD 42019134300).

\section{Criteria for considering studies for this review Types of studies}

Types of studies are randomised clinical trials regardless of publication type, publication status, publication date, and language. We will also include quasi-randomised clinical trials and cluster randomised clinical trials.

\section{Types of participants}

Neonates and infants suspected of or diagnosed with sepsis (as defined by trialists). We will also include neonates and infants (until 3 months of age) suspected of or diagnosed with severe infections such as meningitis, osteomyelitis, endocarditis and necrotizing enterocolitis.

\section{Types of interventions}

We will accept any type of antibiotic or combination of antibiotics (regardless of dose and way of administration) such as the following:

1) Beta-lactam antibiotics

- Narrow-spectrum penicillin antibiotics (e.g. oxacillin, cloxacillin, dicloxacillin, nafcillin, methicillin and penicillin G);

- Broad-spectrum penicillin antibiotics (e.g. ampicillin, amoxicillin, piperacillin, ticarcillin, carbenicillin and mezlocillin);

- Beta-lactam antibiotics with beta-lactamase inhibitors such as clavulanic acid, sulbactam and tazobactam;

- Cephalosporins (e.g. cefazolin, cephalexin, cefuroxime, cefotetan, cefoxitin, ceftriaxone, cefotaxime, ceftazidime, cefepime, cefazolin, ceftobiprole and cefoperazone);

- Carbapenems (e.g. imipenem, meropenem, doripenem and ertapenem) and monobactams (e.g. aztreonam);

Broad-spectrum penicillins, beta-lactam antibiotics with beta-lactamase inhibitors, cephalosporins and carbapenems will be considered as broad-spectrum antibiotics.

2) Combination of beta-lactam with aminoglycoside (e.g. gentamycin)

3) Combination of beta-lactam with glycopeptide (e.g. vancomycin and teicoplanin) 
4) Combination of glycopeptide with aminoglycoside

We plan to assess the following comparisons:

1. Aminoglycoside added to any type of antibiotic versus any type of antibiotic (same antibiotic as in the experimental group).

2. Broad-spectrum beta-lactam antibiotic and aminoglycoside versus narrow-spectrum betalactam antibiotic (as defined in the above) and aminoglycoside (same aminoglycoside as in the experimental group).

3. Beta-lactam antibiotic (as defined in the above) and aminoglycoside versus beta-lactam antibiotic and glycopeptide.

4. Any other used antibiotic regimen (not included in the abovementioned comparisons) versus any other used antibiotic regimen (not included in the abovementioned comparisons).

\section{Co-interventions}

We will accept any co-intervention provided they are intended to be delivered similarly to the experimental and the control group. Assuming no interaction, the effects of the co-interventions will 'even out' in both groups so the possible effects of antibiotics will be reflected in the results.

We will exclude trials assessing treatment of fungal and viral infections.

\section{Types of outcome measures}

The primary outcomes are as follows:

1. All-cause mortality.

2. Proportion of participants with a serious adverse event defined as any untoward medical occurrence that resulted in death; was life threatening; was persistent or led to significant disability, nephrotoxicity, superinfection, need for respiratory support, need for circulatory support or prolonged hospitalisation [122]. As we expect the trialists' reporting of serious adverse events to be heterogeneous and not strictly according to the ICH-GCP recommendations, we will include the event as a serious adverse if the trialists either (1) use the term 'serious adverse event' but not refer to $\mathrm{ICH}-\mathrm{GCP}$ or (2) report the proportion of participants with an event we consider fulfil the ICH-GCP definition (e.g. myocardial infarction or hospitalisation). If several of such events are reported then we will choose the highest proportion reported in each trial to avoid double counting.

The secondary outcomes are as follows:
1. Need for respiratory support defined as the need for respiratory support such as non-invasive ventilation (e.g. CPAP) or invasive ventilation (e.g. respirator)

2. Need for circulatory support defined as the need for circulatory support such as fluid bolus or vasoactive medication (e.g. inotropes or vasopressors).

3. Nephrotoxicity (as defined by the trialist).

4. Presence of moderate-to-severe neurological developmental and sensory impairment (defined as a functional abnormality in the function of the brain, spinal cord, muscles, nerves, eyes or ears or as any significant lag in a child's physical or motor, cognitive, behavioural, emotional or social development, in comparison with other children of the same age and sex within similar environments. If formal evaluation tools were used to assess neurodevelopmental impairment a threshold of -2 standard deviations of the normal will be used. Furthermore, severe brain injury per se is included, such as intraventricular haemorrhage grade 3 and 4 [123, 124] and periventricular leukomalacia.

5. Ototoxicity (as defined by trialist).

6. Necrotizing enterocolitis during or after treatment, Bells criteria 2 [125].

7. Neurological complication defined as either intraventricular haemorrhage [126], psychomotor retardation, or defined by trialist.

All outcomes will be assessed as proportions.

We will use the trial results reported at maximum follow-up. However, if the trialists report results at multiple time points, we will primarily use the results reported at the time point closest to 1 year.

\section{Search methods for identification of studies}

We will use the criteria and standard methods of Cochrane and Cochrane Neonatal (see the Cochrane Neonatal search strategy for specialized register).

\section{Electronic searches}

We will conduct a comprehensive search including Cochrane Central Register of Controlled Trials (CENTRAL, current issue) in The Cochrane Library; MEDLINE via PubMed (1996 to current); MEDLINE via Ovid (1946 to current) Embase (1980 to current); and CINAHL (1982 to current) using search strategies detailed in Additional file 1.

Further searches will be performed in EMBASE for pharmaceutical publications and ZETOC for abstracts of scientific conferences/symposia. References from identified studies were cross-checked for possible additional studies.

We will search clinical trials registries for ongoing or recently completed trials (clinicaltrials.gov; the World 
Health Organization's International Trials Registry and Platform, and the ISRCTN Registry).

We will search all databases from their inception to the present. There will be no restriction by language of publication, and we will arrange for translation where necessary. This will be acknowledged in the 'Acknowledgements' section.

\section{Searching other resources}

We will check reference lists of all relevant primary trials and reviews for additional references. To identify unpublished trials, we will also search clinical trial registers of Europe and the USA, websites of pharmaceutical companies, and websites of the US Food and Drug Administration (FDA) and the European Medicines Agency.

\section{Data collection}

\section{Selection of studies}

Two review authors (SKK and SS) will independently screen titles and abstracts. We will retrieve all relevant full-text study reports/publication and two review authors (SKK and SS) will independently screen the full texts and identify trials for inclusion and identify and record reasons for exclusion of the ineligible studies. We will resolve any disagreement through discussion or, if required, by consulting a third person (JCJ). We will record the selection process in sufficient detail to complete a PRISMA flow diagram [127] and "Characteristics of excluded studies" table.

\section{Data extraction and management}

We will use data collection forms for trial characteristics and outcome data which has been piloted on at least one trial in the review. Two review authors (SKK and SS) will extract trial characteristics from included trials. We will extract the following trials characteristics:

1. Methods - trial design, total duration of the trial, number of trial centres and location, trial setting, bias domain items, withdrawals and date of the trial.

2. Participants-number of participants in each intervention group, mean age, age range, sex, diagnostic criteria, inclusion criteria and exclusion criteria.

3. Interventions-intervention and comparison.

4. Outcomes-primary and secondary outcomes specified and collected and time points reported.

5. Notes-funding for trial, and notable conflicts of interest of trial authors.

Two review authors (SKK and SS) will independently extract outcome data from included trials. We will note in the 'Characteristics of included studies' table if outcome data were not reported in a usable way. We will resolve disagreements by consensus or by involving a third person (JCJ). We will double-check that data are entered correctly by comparing the data presented in the systematic review with the study reports. A second review author (SS) will spot-check study characteristics for accuracy against the trial report.

\section{Assessment of risk of bias in included studies}

Two review authors will independently assess the risk of bias (low, high or unclear) of all included trials using the Cochrane 'Risk of bias' tool [128] for the following domains.

\section{Allocation sequence generation}

- Low risk: If sequence generation was achieved using computer random number generator or a random numbers table. Drawing lots, tossing a coin, shuffling cards and throwing dice were also considered adequate if performed by an independent adjudicator.

- Unclear risk: If the method of randomisation was not specified but the trial was still presented as being randomised.

- High risk: If the allocation sequence was not randomised or only quasi-randomised.

\section{Allocation concealment}

- Low risk: If the allocation of patients was performed by a central independent unit, on-site locked computer, identical-looking numbered sealed envelopes, drug bottles or containers prepared by an independent pharmacist or investigator.

- Uncertain risk: If the trial was classified as randomised but the allocation concealment process was not described.

- High risk: If the allocation sequence was familiar to the investigators who assigned participants.

\section{Blinding of participants and treatment providers}

- Low risk: If the participants and the treatment providers were blinded to intervention allocation, and this was described.

- Uncertain risk: If the procedure of blinding was insufficiently described.

- High risk: If blinding of participants and the treatment providers was not performed.

\section{Blinding of outcome assessment}

- Low risk of bias: If it was mentioned that outcome assessors were blinded and this was described. 
- Uncertain risk of bias: If it was not mentioned if the outcome assessors in the trial were blinded, or the extent of blinding was insufficiently described.

- High risk of bias: If no blinding or incomplete blinding of outcome assessors was performed.

\section{Incomplete outcome data}

- Low risk of bias: If missing data were unlikely to make treatment effects depart from plausible values. This could either be (1) there were no dropouts or withdrawals for all outcomes or (2) the numbers and reasons for the withdrawals and dropouts for all outcomes were clearly stated and could be described as being similar in both groups. Generally, the trial was judged as at a low risk of bias due to incomplete outcome data if dropouts were less than 5\%. However, the $5 \%$ cutoff was not definitive.

- Uncertain risk of bias: If there was insufficient information to assess whether missing data were likely to induce bias on the results.

- High risk of bias: If the results were likely to be biased due to missing data either because the pattern of dropouts could be described as being different in the two intervention groups or the trial used improper methods in dealing with the missing data (e.g. last observation carried forward).

\section{Selective outcome reporting}

- Low risk of bias: If a protocol was published before or at the time the trial was begun and the outcomes specified in the protocol were reported on. If there was no protocol or the protocol was published after the trial was begun, reporting of all-cause mortality and serious adverse events would grant the trial a grade of low risk of bias.

- Uncertain risk of bias: If no protocol was published and the outcomes all-cause mortality and serious adverse events were not reported on.

- High risk of bias: If the outcomes in the protocol were not reported on.

\section{For-profit bias}

- Low risk of bias: If the trial was not financed by a company that might have an interest in a given result.

- Uncertain risk of bias: If there was no description of how the trial was financed.

- High risk of bias: If the trial was financed or have other involvement by a company that might have an interest in a given result.

\section{Other bias}

- Low risk of bias: The trial appeared to be free of other bias domains (e.g. academic) that could put it at risk of bias.

- Unclear risk of bias: The trial may or may not have been free of other domains that could put it at risk of bias.

- High risk of bias: There were other factors in the trial that could put it at risk of bias (e.g. authors have conducted trials on the same topic).

\section{Overall risk of bias}

We assessed overall risk of bias in two groups defined as:

- Low risk of bias: The outcome result was classified as overall 'low risk of bias' only if all of the bias domains described in the above paragraphs were classified as low risk of bias.

- High risk of bias: The outcome result was classified 'high risk of bias' if any of the bias risk domains described in the above excluding 'blinding of participants and personnel' were classified as 'unclear' or 'high risk of bias'.

- Any other bias

Any disagreements will be resolved by discussion or by a third assessor. Our primary conclusions will be based on the results of our primary outcomes at overall low risk of bias. The bias risk assessment enable classification of randomised trials with low risk of bias and high risk of bias. The latter trials tend to overestimate positive intervention effects and underestimate negative effects [129-133].

\section{Data synthesis \\ Measures of treatment effect \\ Dichotomous outcomes}

We will calculate risk ratios (RRs) with 95\% confidence interval (CI) for dichotomous outcomes.

\section{Unit of analysis issues}

The unit of analysis will be the participating infant in individually randomised trials and the neonatal unit (or sub-unit) for cluster-randomised trials. For clusterrandomised trials, we will undertake analyses at the level of the individual while accounting for the clustering in the data using the methods recommended in the Cochrane Handbook for Systematic Reviews of Interventions [134]. 


\section{Dealing with missing data}

We will not impute missing values for any outcomes in our primary analysis. In two of our sensitivity analyses, we will impute data (see 'Sensitivity analysis').

We will contact investigators and trial sponsors in order to verify key trial characteristics and obtain missing numerical outcome data where possible (e.g. when a study is identified as abstract only).

\section{Assessment of heterogeneity}

We will visually inspect forest plots to assess signs of heterogeneity, and we will explore possible heterogeneity in our prespecified subgroup analyses. We will also inspect trial characteristics across trials to identify clinical heterogeneity. We will assess the presence of statistical heterogeneity by the $\chi^{2}$ test (threshold $P<0.10$ ) and measure the quantities of heterogeneity by the $I^{2}$ statistic $[135,136]$. If we detect moderate or high heterogeneity, we plan to explore the possible causes (e.g. differences in study design, participants, interventions or completeness of outcome assessments). Ultimately, we may decide that a meta-analysis should be avoided [134].

\section{Meta-analysis}

We will undertake this meta-analysis according to the recommendations stated in the Cochrane Handbook for Systematic Reviews of Interventions [134]. We will use the statistical software Review Manager 5 [137] provided by Cochrane to analyse data.

We will assess our intervention effects with both random-effects model meta-analyses [138] and fixedeffect model meta-analyses [120, 139]. We will use the more conservative point estimate of the two [120]. We consider 'the more conservative point estimate', the estimate closest to zero effect [120]. If the two estimates are equal, we will use the estimate with the widest CI [120].

We will use two primary outcomes, and, therefore, we will consider a $P$ value of 0.033 or less as the threshold for statistical significance [120]. For all remaining outcomes, we will consider a $P$ value of 0.05 or less as the threshold for statistical significance [120]. We will use the eight-step procedure to assess if the thresholds for significance are crossed [120]. Our primary conclusion will be based on results with low risk of bias [120]. Where data are only available from one trial, we will use Fisher's exact test [140] for dichotomous data.

Where multiple trial arms are reported in a single trial, we will include only the relevant arms. If two comparisons are combined in the same meta-analysis, we will halve the control group to avoid double counting.

\section{Trial sequential analysis}

Traditional meta-analysis (TSA) runs the risk of random errors due to sparse data and repetitive testing of accumulating data when updating reviews. We will therefore perform trial sequential analyses on the outcomes, in order to calculate the required information size and the cumulative $Z$ curve's breach of relevant trial sequential monitoring boundaries [141-147]. We wish to control the risks of type I errors and type II errors. A more detailed description of trial sequential analysis can be found at http://www.ctu.dk/tsa/. We will assess our trial sequential analysis intervention effects with both a random effects model [138] and a fixed-effect model [139]. We will use the more conservative point estimate of the two [120]. The more conservative point estimate will be the estimate closest to zero effect. If the two estimates are similar, we will use the estimate with the widest CI.

For dichotomous outcomes, we will estimate the required information size based on the observed, unweighted proportion of patients with an outcome in the control group (the cumulative proportion of patients with an event in the control groups relative to all patients in the control groups), a relative risk reduction of $20 \%$, an alpha of $3.3 \%$, a beta of $20 \%$ and diversity as suggested by the trials in the meta-analysis.

\section{Meta-bias}

We will use a funnel plot to assess publication bias if ten or more trials are included. We will visually inspect funnel plots to assess the risk of bias. As we plan to report results when analysing dichotomous outcomes using risk ratios, we will not use any test to assess funnel plot asymmetry when analysing dichotomous outcomes [134].

\section{Assessment of bias in conducting the systematic review}

We will conduct the review according to this published protocol and report any deviations from it in the 'Differences between protocol and review' section of the systematic review.

\section{'Summary of findings' table and GRADE}

We will create a 'Summary of Findings' table using each of the prespecified primary outcomes and five prespecified secondary outcomes (respiratory failure, circulatory failure, nephrotoxicity, neurological complication and ototoxicity) at maximum follow-up. We will use the Grading of Recommendations Assessment, Development and Evaluation (GRADE) approach, as outlined in the GRADE Handbook [148] to assess the certainty of the body of evidence for our primary outcomes.

Two authors will independently assess the quality of the evidence for each of the outcomes above. We will consider evidence from randomized controlled trials as high quality but downgrade the evidence one level for serious (or two levels for very serious) limitations based upon the following: design (risk of bias), consistency 
across studies, directness of the evidence, precision of estimates and presence of publication bias. We will assess 'precision of estimates' using TSA [120]. We will use the [149] Guideline Development Tool to create a 'Summary of findings' table to report the quality of the evidence.

The GRADE approach results in an assessment of the quality of a body of evidence in one of four grades:

1. High: We are very confident that the true effect lies close to that of the estimate of the effect.

2. Moderate: We are moderately confident in the effect estimate: the true effect is likely to be close to the estimate of the effect, but there is a possibility that it is substantially different.

3. Low: Our confidence in the effect estimate is limited: the true effect may be substantially different from the estimate of the effect.

4. Very low: We have very little confidence in the effect estimate: the true effect is likely to be substantially different from the estimate of effect.

\section{Subgroup analysis and investigation of heterogeneity}

We plan to carry out the following subgroup analyses for our primary outcomes.

1. High risk of bias trials compared with low risk of bias trials.

2. Trials assessing neonatal sepsis without separation between early and late onset sepsis compared to separation at either $48 \mathrm{~h}, 72 \mathrm{~h}$ or 7 days.

3. Gestational age: term ( $\geq 37$ weeks) compared with preterm

4. Trials from high-income countries compared with trials from low- and middle-income countries as defined by the World Bank [150]

5. Route of administration such as either oral, intramuscular or intravenous

We will use the formal test for subgroup interactions in Review Manager [137].

\section{Sensitivity analysis}

To assess the potential impact of the missing data, we will perform the two following sensitivity analyses on the primary outcomes.

- 'Best-worst-case' scenario: we will assume that all participants lost to follow-up in the experimental group have survived and had no serious adverse event, and all those participants with missing outcomes in the control group have not survived and have had a serious adverse event.
- 'Worst-best-case' scenario. we will assume that all participants lost to follow-up in the experimental group have not survived and have had a serious adverse event and that all those participants lost to follow-up in the control group had survived and had no serious adverse event.

We will present results of both scenarios in our review.

Other post-hoc sensitivity analyses might be warranted if unexpected clinical or statistical heterogeneity is identified during the analysis of the review results [120].

\section{Discussion}

This protocol has several methodological strengths. First, this will be a systematic review that includes neonates with both early and late onset neonatal sepsis, which increases the statistical power and may lead to conclusive results. Second, our methodology is described in detail in this protocol which will be published before the literature search is initiated. Third, we will conduct the review based on the Cochrane Handbook and findings and recommendation of additional methodological studies [120, 135]. Hence, we will systematically assess the risks of systematic errors via bias risk assessments, and we will conduct trial sequential analyses and properly adjust our thresholds for statistical significance to control the risks of random error. This adds further robustness to our results and hence to our conclusions [151]. Forth, we will use our systematic eight step procedure to assess if the thresholds for statistical and clinical significance are crossed [120].

Neonatal sepsis and sepsis among infants are syndromes with high clinical heterogeneity and without internationally agreed upon diagnostic criteria. The underlying bacteria causing sepsis are expected to differ in the different trials as we include trials regardless of onset (in the neonatal period) and location (country). The doses and length of therapy of the antibiotic regimens might also differ between trials, and the trials we will include will possibly use different inclusion criteria. Therefore, the clinical heterogeneity between the trials might be relatively high. This expected heterogeneity may cause significant differences in mortality rates among different trials. In addition, there might be substantial differences in the types of serious adverse events the trials report, which may compromise the validity of the serious adverse event outcome. Also, it is possible that there exist temporospatial differences in other elements of sepsis treatment that may lead to potential differences in results among different trials. We plan to carefully consider these potential limitations in the main publication. We do not expect to include a large number of relevant trials, which potentially will limit the statistical power of this review. 


\section{Supplementary information}

Supplementary information accompanies this paper at https://doi.org/10. 1186/s13643-019-1207-1.

Additional file 1. Medline via Ovid Search Strategy.

\section{Abbreviations}

MRSA: Methicillin-resistant Staphylococcus aureus; NICU: Neonatal intensive care unit; PROM: Preterm rupture of the membrane

\section{Authors' contributions}

Conceiving the review: SKK. Co-ordinating the review: SKK. Undertaking manual searches: SKK. Screening search results: SKK and SS. Organizing retrieval of papers: SKK and SS. Screening retrieved papers against inclusion criteria: SKK and SS. Appraising quality of papers: SKK and SS. Abstracting data from papers: SKK and SS. Writing to authors of papers for additional information: SKK and SS. Providing additional data about papers: SKK and SS. Obtaining and screening data on unpublished studies: SKK and SS. Data management for the review: SKK and SS. Entering data into Review Manager: SKK and SS. RevMan statistical data: SKK, SS, CG, ULT and JCJ. Other statistical analysis not using RevMan: SKK and SS. Interpretation of data: SKK, SS, CG, ULT and JCJ. Writing the review: SKK and SS. Securing funding for the review: SKK, CG and JCJ. Guarantor for the review (one author): SKK. Person responsible for reading and checking review before submission: SKK, SS, CG, ULT and JCJ. All authors read and approved the final manuscript.

\section{Funding}

The author team has not received any funding as support for the protocol.

\section{Competing interests}

The authors declare that they have no competing interests.

\section{Author details}

${ }^{1}$ Copenhagen Trial Unit, Centre for Clinical Intervention Research, Department 7812, Copenhagen University Hospital, Rigshospitalet, Copenhagen, Denmark. ²Department of Neonatology, Copenhagen University Hospital, Rigshospitalet, Copenhagen, Denmark. ${ }^{3}$ Department of Regional Health Research, The Faculty of Health Sciences, University of Southern Denmark, Odense, Denmark. ${ }^{4}$ Department of Cardiology, Holbæk Hospital, Holbæk, Denmark.

\section{Received: 4 July 2019 Accepted: 22 October 2019}

Published online: 05 December 2019

\section{References}

1. Bakhuizen SE, de Haan TR, Teune MJ, van Wassenaer-Leemhuis AG, van der Heyden JL, van der Ham DP, et al. Meta-analysis shows that infants who have suffered neonatal sepsis face an increased risk of mortality and severe complications. Acta Paediatr. 2014;103(12):1211-8.

2. Camacho-Gonzalez A, Spearman PW, Stoll BJ. Neonatal infectious diseases: evaluation of neonatal sepsis. Pediatr Clin North Am. 2013;60(2):367-89.

3. Wynn JL. Defining neonatal sepsis. Current Opinion in Pediatrics. 2016;28(2):135-40.

4. Wynn JL, Wong HR, Shanley TP, Bizzarro MJ, Saiman L, Polin RA. Time for a neonatal-specific consensus definition for sepsis. Pediatr Crit Care Med. 2014;15(6):523-8.

5. Morris JM, Roberts CL, Bowen JR, Patterson JA, Bond DM, Algert CS, et al. Immediate delivery compared with expectant management after preterm pre-labour rupture of the membranes close to term (PPROMT trial): a randomised controlled trial. Lancet. 2016;387(10017):444-52.

6. Shane AL, Sanchez PJ, Stoll BJ. Neonatal sepsis. Lancet. 2017;390(10104): 1770-80.

7. Karunasekera KA, Pathirana D. A preliminary study on neonatal septicaemia in a tertiary referral hospital paediatric unit. Ceylon Medical Journal. 1999; 44(2):81-6.

8. Lim NL, Wong YH, Boo NY, Kasim MS, Chor CY. Bacteraemic infections in a neonatal intensive care unit--a nine-month survey. Med J Malaysia. 1995; 50(1):59-63.

9. Moreno MT, Vargas S, Poveda R, Sáez-Llorens X. Neonatal sepsis and meningitis in a developing Latin American country. Pediatric Infectious Disease Journal. 1994;13(6):516-20.
10. Robillard PY, Nabeth P, Hulsey TC, Sergent MP, Périanin J, Janky E. Neonatal bacterial septicemia in a tropical area. Four-year experience in Guadeloupe (French West Indies). Acta Paediatr. 1993;82(8):687-9.

11. Tallur SS, Kasturi AV, Nadgir SD, Krishna BV. Clinico-bacteriological study of neonatal septicemia in Hubli. Indian J Pediatr. 2000;67(3):169-74.

12. The Who Young Infants Study Group. Clinical prediction of serious bacterial infections in young infants in developing countries. Pediatric Infectious Disease Journal. 1999;18(10 Suppl):S23-31.

13. Lawn JE, Cousens S, Zupan J. Lancet Neonatal Survival Steering T. 4 million neonatal deaths: when? Where? Why? Lancet. 2005;365(9462):891-900.

14. Liu L, Johnson HL, Cousens S, Perin J, Scott S, Lawn JE, et al. Global, regional, and national causes of child mortality: an updated systematic analysis for 2010 with time trends since 2000. Lancet. 2012;379(9832):2151-61.

15. Kabwe M, Tembo J, Chilukutu L, Chilufya M, Ngulube F, Lukwesa C, et al. Etiology, antibiotic resistance and risk factors for neonatal sepsis in a large referral center in Zambia. Pediatr Infect Dis J. 2016;35(7):e191-8.

16. Weston EJ, Pondo T, Lewis MM, Martell-Cleary P, Morin C, Jewell B, et al. The burden of invasive early-onset neonatal sepsis in the United States, 20052008. Pediatric Infectious Disease Journal. 2011;30(11):937-41.

17. Benjamin DKJ, Stoll BJ, Fanaroff AA, McDonald SA, Oh W, Higgins RD, et al. Neonatal candidiasis among extremely low birth weight infants: risk factors, mortality rates, and neurodevelopmental outcomes at 18 to 22 months. Pediatrics. 2006;117(1):84-92.

18. Klinger G, Levy I, Sirota L, Boyko V, Lerner-Geva L, Reichman B, et al. Outcome of early-onset sepsis in a national cohort of very low birth weight infants. Pediatrics. 2010;125(4):e736-e40.

19. Schlapbach $L$, Aebischer M, Adams M, Natalucci G, Bonhoeffer J, Latzin P, et al. Impact of sepsis on neurodevelopmental outcome in a Swiss National Cohort of extremely premature infants. Pediatrics. 2011;128(2):e348-e57.

20. Bizzarro MJ, Dembry LM, Baltimore RS, Gallagher PG. Changing patterns in neonatal Escherichia coli sepsis and ampicillin resistance in the era of intrapartum antibiotic prophylaxis. Pediatrics. 2008;121(4):689-96.

21. Manan MM, Ibrahim NA, Aziz NA, Zulkifly HH, Al-Worafi YMA, Long CM. Empirical use of antibiotic therapy in the prevention of early onset sepsis in neonates: a pilot study. Arch Med Sci. 2016;12(3):603-13.

22. Shane AL, Stoll BJ. Recent developments and current issues in the epidemiology, diagnosis, and management of bacterial and fungal neonatal sepsis. American Journal of Perinatology. 2013;30(2):131-41.

23. Shane AL, Stoll BJ. Neonatal sepsis: progress towards improved outcomes. J Infect. 2014;68(Suppl 1):S24-32.

24. Tripathi N, Cotten CM, Smith PB. Antibiotic use and misuse in the neonatal intensive care unit. Clinics in Perinatology. 2012;39(1):61-8.

25. Zaidi AKM, Thaver D, Ali SA, Khan TA. Pathogens associated with sepsis in newborns and young infants in developing countries. Pediatric Infectious Disease Journal. 2009;28(1 Suppl):S10-58.

26. Zea-Vera A, Ochoa TJ. Challenges in the diagnosis and management of neonatal sepsis. Journal of Tropical Pediatrics. 2015;61(1):1-13.

27. Shah BA, Padbury JF. Neonatal sepsis: an old problem with new insights. Virulence. 2014;5(1):170-8.

28. Park SE. Prevention of neonatal group B streptococcal disease. Infection \& chemotherapy. 2013:45(3):343-5.

29. Stoll BJ, Hansen N, Fanaroff AA, Wright LL, Carlo WA, Ehrenkranz RA, et al. Changes in pathogens causing early-onset sepsis in very-low-birth-weight infants. N Engl J Med. 2002;347(4):240-7.

30. Boghossian NS, Page GP, Bell EF, Stoll BJ, Murray JC, Cotten CM, et al. Lateonset sepsis in very low birth weight infants from singleton and multiplegestation births. J Pediatr. 2013;162(6):1120-1124, 4.e1.

31. Dong Y, Speer CP. Late-onset neonatal sepsis: recent developments. Archives of Disease in Childhood Fetal and Neonatal Edition. 2015;100(3): F257-63.

32. Stoll BJ, Gordon T, Korones SB, Shankaran S, Tyson JE, Bauer CR, et al. Lateonset sepsis in very low birth weight neonates: a report from the National Institute of Child Health and Human Development Neonatal Research Network. Journal of Pediatrics. 1996;129(1):63-71.

33. Kumar SKM, Bhat BV. Distinct mechanisms of the newborn innate immunity Immunology Letters. 2016;173:42-54.

34. Kan B, Razzaghian HR, Lavoie PM. An immunological perspective on neonatal sepsis. Trends in Molecular Medicine. 2016;22(4):290-302.

35. Rogosch T, Kerzel S, Hoss K, Hoersch G, Zemlin C, Heckmann M, et al. IgA response in preterm neonates shows little evidence of antigen-driven selection. J Immunol. 2012;189(11):5449-56. 
36. Walker JC, MAJC S, EFA G, TAJ A, Leuvenink J, de Vries E. Development of lymphocyte subpopulations in preterm infants. Scandinavian Journal of Immunology. 2011;73(1):53-8.

37. Ygberg S, Nilsson A. The developing immune system - from foetus to toddler. Acta Paediatr. 2012;101(2):120-7.

38. Zemlin M, Hoersch G, Zemlin C, Pohl-Schickinger A, Hummel M, Berek C, et al. The postnatal maturation of the immunoglobulin heavy chain lgG repertoire in human preterm neonates is slower than in term neonates. Journal of Immunology. 2007;178(2):1180-8.

39. Stoll BJ, Hansen NI, Sánchez PJ, Faix RG, Poindexter BB, Van Meurs KP, et al. Early onset neonatal sepsis: the burden of group B Streptococcal and E. coli disease continues. Pediatrics. 2011;127(5):817-26.

40. Naher BS, Mannan MA, Noor K, Shahiddullah M. Role of serum procalcitonin and $\mathrm{C}$-reactive protein in the diagnosis of neonatal sepsis. Bangladesh Medical Research Council bulletin. 2011:37(2):40-6.

41. Leal YA, Álvarez-Nemegyei J, Velázquez JR, Rosado-Quiab U, DiegoRodríguez N, Paz-Baeza E, et al. Risk factors and prognosis for neonatal sepsis in southeastern Mexico: analysis of a four-year historic cohort followup. BMC Pregnancy Childbirth. 2012;12:48.

42. Stoll BJ, Hansen N, Fanaroff AA, Wright LL, Carlo WA, Ehrenkranz RA, et al. Late-onset sepsis in very low birth weight neonates: the experience of the NICHD Neonatal Research Network. Pediatrics. 2002; 110(2 Pt 1):285-91.

43. Tröger B, Göpel W, Faust K, Müller T, Jorch G, Felderhoff-Müser U, et al. Risk for late-onset blood-culture proven sepsis in very-low-birth weight infants born small for gestational age: a large multicenter study from the German Neonatal Network. Pediatr Infect Dis J. 2014;33(3):238-43.

44. Tsai MH, Hsu JF, Chu SM, Lien R, Huang HR, Chiang MC, et al. Incidence, clinical characteristics and risk factors for adverse outcome in neonates with late-onset sepsis. Pediatric Infectious Disease Journal. 2014;33(1):e7-e13.

45. Deutschman CS, Tracey KJ. Sepsis: current dogma and new perspectives. Immunity. 2014;40(4):463-75.

46. Singer M, Deutschman CS, Seymour CW, Shankar-Hari M, Annane D, Bauer $M$, et al. The third international consensus definitions for sepsis and septic shock (sepsis-3). JAMA. 2016;315(8):801-10.

47. Seale AC, Obiero CW, Berkley JA. Rational development of guidelines for management of neonatal sepsis in developing countries. Curr Opin Infect Dis. 2015;28(3):225-30.

48. World Health Organization. Pocket book of hospital care for children: Second edition. Guidelines for the management of common childhood illnesses. http://appswhoint/iris/bitstream/10665/81170/1/9789241548373_ engpdf?ua $=12017$.

49. National Institute for $\mathrm{H}$, Clinical Excellence. Neonatal infection (early onset) antibiotics for prevention and treatment. https://wwwniceorguk/guidance/ cg1492012.

50. Rubin LG, Sánchez PJ, Siegel J, Levine G, Saiman L, Jarvis WR, et al. Evaluation and treatment of neonates with suspected late-onset sepsis: a survey of neonatologists' practices. Pediatrics. 2002;110(4):e42

51. National Institute for $\mathrm{H}$, Clinical Excellence. Neonatal infection (early onset) antibiotics for prevention and treatment. https://wwwniceorguk/guidance/ cg149/evidence/evidence-update-pdf-188168797. 2014.

52. Golan DE, Tashlian AH, Amstrong EJ, Armstrong AW. Principles of pharmacology. Philadelphia: Lippincott Williams \& Wilkins. 2011;2011.

53. Katzung BG, Masters SB, Trevor AJ. Basic and clinical pharmacology: McGraw-Hill Medical Publishing Division; 2009; 2009.

54. Vergnano S, Menson E, Kennea N, Embleton N, Russell AB, Watts T, et al. Neonatal infections in England: the NeonIN surveillance network. Archives of Disease in Childhood Fetal and Neonatal Edition. 2011;96(1):F9-F14.

55. Cortese F, Scicchitano P, Gesualdo M, Filaninno A, De Giorgi E, Schettini F, et al. Early and late infections in newborns: where do we stand? A review. Pediatrics and Neonatology. 2016;57(4):265-73.

56. Muller-Pebody B, Johnson AP, Heath PT, Gilbert RE, Henderson KL, Sharland $M$, et al. Empirical treatment of neonatal sepsis: are the current guidelines adequate? Archives of Disease in Childhood Fetal and Neonatal Edition. 2011;96(1):F4-8.

57. Vergnano S, Sharland M, Kazembe P, Mwansambo C, Heath PT. Neonatal sepsis: an international perspective. Archives of Disease in Childhood Fetal and Neonatal Edition. 2005;90(3):F220-F4.

58. Cantey J, Wozniak PS, Sánchez PJ. Prospective surveillance of antibiotic use in the neonatal intensive care unit: results from the SCOUT study. Pediatric Infectious Disease Journal. 2015;34(3):267-72.
59. Clark RH, Bloom BT, Spitzer AR, Gerstmann DR. Empiric use of ampicillin and cefotaxime, compared with ampicillin and gentamicin, for neonates at risk for sepsis is associated with an increased risk of neonatal death. Pediatrics. 2006;117(1):67-74.

60. Tzialla C, Borghesi A, Serra G, Stronati M, Corsello G. Antimicrobial therapy in neonatal intensive care unit. Ital J Pediatr. 2015:41:27.

61. Bizzarro MJ, Shabanova V, Baltimore RS, Dembry LM, Ehrenkranz RA, Gallagher PG. Neonatal sepsis 2004-2013: the rise and fall of coagulasenegative staphylococci. J Pediatr. 2015;166(5):1193-9.

62. Marchant EA, Boyce GK, Sadarangani M, Lavoie PM. Neonatal sepsis due to coagulase-negative staphylococci. Clinical \& developmental immunology. 2013;2013:586076-

63. Stockmann C, Spigarelli MG, Campbell SC, Constance JE, Courter JD, Thorell EA, et al. Considerations in the pharmacologic treatment and prevention of neonatal sepsis. Paediatric drugs. 2014;16(1):67-81.

64. Dellinger RP, Levy MM, Rhodes A, Annane D, Gerlach H, Opal SM, et al. Surviving Sepsis Campaign: international guidelines for management of severe sepsis and septic shock, 2012. Intensive Care Medicine. 2013;39(2): $165-228$.

65. Gaieski DF, Mikkelsen ME, Band RA, Pines JM, Massone R, Furia FF, et al. Impact of time to antibiotics on survival in patients with severe sepsis or septic shock in whom early goal-directed therapy was initiated in the emergency department. Crit Care Med. 2010;38(4):1045-53.

66. Cohen ML. Epidemiology of drug resistance: implications for a postantimicrobial era. Science (New York, NY). 1992;257(5073):1050-5.

67. Foster KR, Grundmann H. Do we need to put society first? The potential for tragedy in antimicrobial resistance. PLoS medicine. 2006;3(2):e29-e.

68. Huynh BT, Padget M, Garin B, Delarocque-Astagneau E, Guillemot D. Birdy study group. Bacterial neonatal sepsis and antibiotic resistance in lowincome countries. Lancet. 2016:387(10018):533-4.

69. Kunin CM, Johansen KS, Worning AM, Daschner FD. Report of a symposium on use and abuse of antibiotics worldwide. Reviews of infectious diseases. 1990;12(1):12-9

70. Jr MGJ. Do intensive hospital antibiotic control programs prevent the spread of antibiotic resistance? Infection Control and Hospital Epidemiology 1994:15(7):478-83.

71. Murray BE. Can antibiotic resistance be controlled? New England Journal of Medicine. 1994;330(17):1229-30.

72. Sáez-Llorens X, Castrejon de Wong MM, Castano E, De Suman O, De Moros $D$, De Atencio I. Impact of an antibiotic restriction policy on hospital expenditures and bacterial susceptibilities: a lesson from a pediatric institution in a developing country. Pediatr Infect Dis J. 2000;19(3):200-6.

73. Breurec S, Bouchiat C, Sire JM, Moquet O, Bercion R, Cisse MF, et al. High third-generation cephalosporin resistant Enterobacteriaceae prevalence rate among neonatal infections in Dakar, Senegal. BMC Infectious Diseases. 2016; 16(1):587.

74. Isaacs D. Australasian Study Group For Neonatal Infections. A ten year, multicentre study of coagulase negative staphylococcal infections in Australasian neonatal units. Archives of Disease in Childhood Fetal and Neonatal Edition. 2003;88(2):F89-93.

75. May M, Daley AJ, Donath S, Isaacs D. Australasian Study Group for Neonatal Infections. Early onset neonatal meningitis in Australia and New Zealand, 1992-2002. Archives of Disease in Childhood Fetal and Neonatal Edition. 2005;90(4):F324-F7.

76. Stoll BJ, Hansen N. Infections in VLBW infants: studies from the NICHD Neonatal Research Network. Semin Perinatol. 2003;27(4):293-301.

77. Stoll BJ, Hansen NI, Higgins RD, Fanaroff AA, Duara S, Goldberg R, et al. Very low birth weight preterm infants with early onset neonatal sepsis: the predominance of gram-negative infections continues in the National Institute of Child Health and Human Development Neonatal Research Network, 2002-2003. Pediatr Infect Dis J. 2005;24(7):635-9.

78. Khatua SP, Das AK, Chatterjee BD, Khatua S, Ghose B, Saha A. Neonatal septicemia. Indian J Pediatr. 1986;53(4):509-14.

79. Tessin I, Trollfors B, Thiringer K. Incidence and etiology of neonatal septicaemia and meningitis in western Sweden 1975-1986. Acta Paediatr Scand. 1990;79(11):1023-30.

80. Vesikari T, Janas M, Grönroos P, Tuppurainen N, Renlund M, Kero P, et al. Neonatal septicaemia. Archives of Disease in Childhood. 1985:60(6):542-6.

81. Zaidi AKM, Huskins WC, Thaver D, Bhutta ZA, Abbas Z, Goldmann DA. Hospital-acquired neonatal infections in developing countries. Lancet. 2005; 365(9465):1175-88. 
82. Cotton MF, Wasserman E, Pieper CH, Theron DC, van Tubbergh D, Campbell $\mathrm{G}$, et al. Invasive disease due to extended spectrum beta-lactamaseproducing Klebsiella pneumoniae in a neonatal unit: the possible role of cockroaches. Journal of Hospital Infection. 2000;44(1):13-7.

83. Gonzalez-Vertiz A, Alcantar-Curiel D, Cuauhtli M, Daza C, Gayosso C, Solache $\mathrm{G}$, et al. Multiresistant extended-spectrum beta-lactamase-producing Klebsiella pneumoniae causing an outbreak of nosocomial bloodstream infection. Infection Control and Hospital Epidemiology. 2001;22(11):723-5.

84. Shenoy S, Hegde A, Dominic SRM, Kamath S, Arvind N. An outbreak of extended spectrum beta-lactamase producing Klebsiella pneumoniae in a neonatal intensive care unit. Indian Journal of Pathology \& Microbiology. 2007;50(3):669-70.

85. Mrvos R, Pummer TL, Krenzelok EP. Amoxicillin renal toxicity: how often does it occur? Pediatr Emerg Care. 2013;29(5):641-3.

86. Gillies M, Ranakusuma A, Hoffmann T, Thorning S, McGuire T, Glasziou P, et al. Common harms from amoxicillin: a systematic review and metaanalysis of randomized placebo-controlled trials for any indication. CMAJ. 2015;187(1):E21-31.

87. Huth ME, Ricci AJ, Cheng AG. Mechanisms of aminoglycoside ototoxicity and targets of hair cell protection. International Journal of Otolaryngology. 2011;2011:937861-

88. Jackson GG, Arcieri G. Ototoxicity of gentamicin in man: a survey and controlled analysis of clinical experience in the United States. Journal of Infectious Diseases. 1971;124(Suppl):S130-S7.

89. Mattie $\mathrm{H}$, Craig WA, Pechère JC. Determinants of efficacy and toxicity of aminoglycosides. Journal of Antimicrobial Chemotherapy. 1989;24(3):281-93.

90. McGlone A, Cranswick N. Evidence behind the WHO guidelines: Hospital care for children: what is the evidence of safety of gentamicin use in children? Journal of Tropical Pediatrics. 2008;54(5):291-3.

91. Mingeot-Leclercq MP, Tulkens PM. Aminoglycosides: nephrotoxicity. Antimicrobial Agents and Chemotherapy. 1999;43(5):1003-12.

92. Musiime GM, Seale AC, Moxon SG, Lawn JE. Risk of gentamicin toxicity in neonates treated for possible severe bacterial infection in low- and middleincome countries: Systematic Review. Trop Med Int Health. 2015;20(12): 1593-606.

93. Schultze RG, Winters RE, Kauffman H. Possible nephrotoxicity of gentamicin. Journal of Infectious Diseases. 1971;124(Suppl):S145-S7.

94. Selimoglu E. Aminoglycoside-induced ototoxicity. Curr Pharm Des. 2007; 13(1):119-26.

95. Wargo KA, Edwards JD. Aminoglycoside-induced nephrotoxicity. Journal of Pharmacy Practice. 2014;27(6):573-7.

96. Rybak MJ, Lomaestro BM, Rotschafer JC, Rc M Jr, Craig WA, Billeter M, et al. Therapeutic monitoring of vancomycin in adults summary of consensus recommendations from the American Society of Health-System Pharmacists, the Infectious Diseases Society of America, and the Society of Infectious Diseases Pharmacists. Pharmacotherapy. 2009;29(11):1275-9.

97. Farber BF, Moellering RC. Retrospective study of the toxicity of preparations of vancomycin from 1974 to 1981. Antimicrobial Agents and Chemotherapy. 1983;23(1):138-41.

98. Bisrat H, Michael N, Anthony W. Frequency of nephrotoxicity with vancomycin and aminoglycoside antibiotic therapy. Hospital Pharmacy. 1999;34(12):1417-20.

99. Salama S, Rotstein C, Mandell L. A multidisciplinary hospital-based antimicrobial use program: Impact on hospital pharmacy expenditures and drug use. Can J Infect Dis. 1996;7(2):104-9.

100. Sorrell TC, Collignon PJ. A prospective study of adverse reactions associated with vancomycin therapy. J Antimicrob Chemother. 1985;16(2):235-41.

101. Cotten CM, McDonald S, Stoll B, Goldberg RN, Poole K. Benjamin Dk Jr. The association of third-generation cephalosporin use and invasive candidiasis in extremely low birth-weight infants. Pediatrics. 2006;118(2):717-22.

102. Filioti J, Spiroglou K, Roilides E. Invasive candidiasis in pediatric intensive care patients: epidemiology, risk factors, management, and outcome. Intensive care medicine. 2007;33(7):1272-83.

103. Spiliopoulou A, Dimitriou G, Jelastopulu E, Giannakopoulos I, Anastassiou ED, Christofidou M. Neonatal intensive care unit candidemia: epidemiology, risk factors, outcome, and critical review of published case series. Mycopathologia. 2012;173(4):219-28.

104. Waksman SA. What is an antibiotic or an antibiotic substance? Mycologia. 1947;39(5):565-9.

105. Bérdy J. Bioactive microbial metabolites. Journal of Antibiotics. 2005; 58(1):1-26.
106. Allan JD, Moellering RC. Management of infections caused by gramnegative bacilli: the role of antimicrobial combinations. Review of Infectious Diseases. 1985;7(Suppl 4):S559-S71.

107. Milatovic D, Braveny I. Development of resistance during antibiotic therapy. European Journal of Clinical Microbiology. 1987;6(3):234-44.

108. Tamma PD, Cosgrove SE, Maragakis LL. Combination therapy for treatment of infections with gram-negative bacteria. Clinical Microbiology Reviews. 2012;25(3):450-70.

109. Ibrahim EH, Sherman G, Ward S, Fraser VJ, Kollef MH. The influence of inadequate antimicrobial treatment of bloodstream infections on patient outcomes in the ICU setting. Chest. 2000;118(1):146-55.

110. Leibovici L, Shraga I, Drucker M, Konigsberger H, Samra Z, Pitlik SD. The benefit of appropriate empirical antibiotic treatment in patients with bloodstream infection. Journal of Internal Medicine. 1998;244(5):379-86.

111. Paul M, Shani V, Muchtar E, Kariv G, Robenshtok E, Leibovici L. Systematic review and meta-analysis of the efficacy of appropriate empiric antibiotic therapy for sepsis. Antimicrobial Agents and Chemotherapy. 2010;54(11): 4851-63.

112. Clark RH, Bloom BT, Spitzer AR, Gerstmann DR. Reported medication use in the neonatal intensive care unit: data from a large national data set. Pediatrics. 2006;117(6):1979-87.

113. Bedford Russell AR, Kumar R. Early onset neonatal sepsis: diagnostic dilemmas and practical management. Archives of Disease in Childhood Fetal and Neonatal Edition. 2015;100(4):F350-4.

114. Luck S, Torny M, d'Agapeyeff K, Pitt A, Heath P, Breathnach A, et al. Estimated early-onset group B streptococcal neonatal disease. Lancet. 2003; 361(9373):1953-4.

115. Cordero L, Ayers LW. Duration of empiric antibiotics for suspected earlyonset sepsis in extremely low birth weight infants. Infection Control and Hospital Epidemiology. 2003;24(9):662-6.

116. Cotten CM, Taylor S, Stoll B, Goldberg RN, Hansen NI, Sánchez PJ, et al. Prolonged duration of initial empirical antibiotic treatment is associated with increased rates of necrotizing enterocolitis and death for extremely low birth weight infants. Pediatrics. 2009;123(1):58-66.

117. Kuppala VS, Meinzen-Derr J, Morrow AL, Schibler KR. Prolonged initial empirical antibiotic treatment is associated with adverse outcomes in premature infants. J Pediatr. 2011;159(5):720-5.

118. Gordon A, Jeffery HE. Antibiotic regimens for suspected late onset sepsis in newborn infants. John Wiley \& Sons, Ltd: Cochrane Database of Systematic Reviews; 2005.

119. Mtitimila El, Cooke RWI. Antibiotic regimens for suspected early neonatal sepsis. Cochrane Database Syst Rev. 2004;4:CD004495.

120. Jakobsen JC, Wetterslev J, Winkel P, Lange T, Gluud C. Thresholds for statistical and clinical significance in systematic reviews with meta-analytic methods. BMC medical research methodology. 2014;14:120.

121. Shamseer L, Moher D, Clarke M, Ghersi D, Liberati A, Petticrew M, et al. Preferred reporting items for systematic review and meta-analysis protocols (PRISMA-P) 2015: elaboration and explanation. Bmj. 2015;350: g7647.

122. Hubbard WK, editor International Conference on Harmonisation; Good Clinical Practice: Consolidated Guideline; Availability. the Federal Register; 1997 1997: Barnett International/PAREXEL.

123. Papile LA, Burstein J, Burstein R, Koffler H. Incidence and evolution of subependymal and intraventricular hemorrhage: a study of infants with birth weights less than 1,500 gm. - PubMed - NCBI. J Pediatrics. 1978;92(4): 529-34.

124. Volpe JJ. Intracranial hemorrhage: germinal matrix intraventricular hemorrhage. Neurology of the Newborn. 5 ed 2008. p. 517-588.

125. Bell MJ, Ternberg JL, Feigin RD, Keating JP, Marshall R, Barton L, et al. Neonatal necrotizing enterocolitis. Therapeutic decisions based upon clinical staging. Annals of Surgery. 1978;187(1):1-7.

126. Volpe JJ. Intracranial hemorrhage: germinal matrix intraventricular hemorrhage; 2008. p. 517-88.

127. Moher D, Liberati A, Tetzlaff J, Altman DG. Preferred reporting items for systematic reviews and meta-analyses: the PRISMA statement. Journal of Clinical Epidemiology. 2009;62(10):1006-12--12.

128. Higgins JPT, Green S. Cochrane handbook for systematic reviews of interventions version 5.2.0 (updated June 2017). The Cochrane Collaboration, 2017. Available from trainingcochraneorg/handbook.

129. Gluud LL. Bias in clinical intervention research. American journal of epidemiology. 2006;163(6):493-501. 
130. Kjaergard LL, Villumsen J, Gluud C. Reported methodologic quality and discrepancies between large and small randomized trials in meta-analyses. Annals of Internal Medicine. 2001;135(11):982-9.

131. Moher D, Pham B, Jones A, Cook DJ, Jadad AR, Moher M, et al. Does quality of reports of randomised trials affect estimates of intervention efficacy reported in meta-analyses? Lancet. 1998;352(9128):609-13.

132. Savovic J, Jones HE, Altman DG, Harris RJ, Juni P, Pildal J, et al. Influence of reported study design characteristics on intervention effect estimates from randomized, controlled trials. Annals of Internal Medicine. 2012;157(6):429-38.

133. Wood L, Egger M, Gluud LL, Schulz KF, Juni P, Altman DG, et al. Empirical evidence of bias in treatment effect estimates in controlled trials with different interventions and outcomes: meta-epidemiological study. BMJ (Clinical Research Ed). 2008;336(7644):601-5.

134. Higgins JPT, Green S. Cochrane Handbook for Systematic Reviews of Interventions Version 5.1 [updated March 2011]. The Cochrane Collaboration, 2011. Available from wwwcochrane-handbookorg.

135. Higgins JP, Thompson SG. Quantifying heterogeneity in a meta-analysis. Statistics in Medicine. 2002;21(11):1539-58.

136. Higgins JP, Thompson SG, Deeks JJ, Altman DG. Measuring inconsistency in meta-analyses. BMJ (Clinical Research Ed). 2003;327(7414):557-60.

137. Review Manager 5 (RevMan 5). 2014.

138. DerSimonian R, Laird N. Meta-analysis in clinical trials. Controlled Clinical Trials. 1986;7(3):177-88.

139. DeMets DL. Methods of combining randomized trials: strength and limitations. Statistics in Medicine. 1987;6(3):341-50.

140. Fisher RA. On the interpretation of $x 2$ from contingency tables, and the calculation of P. Journal of the Royal Statistical Society. 1922;85(1):87-94.

141. Brok J, Thorlund K, Gluud C, Wetterslev J. Trial sequential analysis reveals insufficient information size and potentially false positive results in many meta-analyses. Journal of Clinical Epidemiology. 2008;61(8):763-9.

142. Brok J, Thorlund K, Wetterslev J, Gluud C. Apparently conclusive metaanalyses may be inconclusive--Trial sequential analysis adjustment of random error risk due to repetitive testing of accumulating data in apparently conclusive neonatal meta-analyses. International Journal of Epidemiology. 2009;38(1):287-98.

143. Thorlund K, Devereaux PJ, Wetterslev J, Guyatt G, loannidis JP, Thabane L, et al. Can trial sequential monitoring boundaries reduce spurious inferences from meta-analyses? International Journal of Epidemiology. 2009;38(1):276-86.

144. Thorlund K, Engstrøm J, Wetterslev J, Brok J, Imberger G, Gluud C. User manual for Trial Sequential Analysis (TSA), 2011. ctudk/tsa/files/tsa_ manualpdf. 2015.

145. Wetterslev J, Jakobsen JC, Gluud C. Trial Sequential Analysis in systematic reviews with meta-analysis. BMC medical research methodology. 2017;17(1):39.

146. Wetterslev J, Thorlund K, Brok J, Gluud C. Trial sequential analysis may establish when firm evidence is reached in cumulative meta-analysis. Journal of Clinical Epidemiology. 2008;61(1):64-75.

147. Wetterslev J, Thorlund K, Brok J, Gluud C. Estimating required information size by quantifying diversity in random-effects model meta-analyses. BMC Medical Research Methodology. 2009;9:86.

148. Schünemann H, Brożek J, Guyatt G, Oxman A, editor. Handbook for grading the quality of evidence and the strength of recommendations using the GRADE approach (updated October 2013). GRADE Working Group, 2013. Available from gdtguidelinedevelopmentorg/app/handbook/handbookhtml.

149. GRADEpro GDT. Hamilton (ON): GRADE Working Group: McMaster University; 2014

150. Alm B, Erdes L, Möllborg P, Pettersson R, Norvenius SG, Aberg N, et al. Neonatal antibiotic treatment is a risk factor for early wheezing. Pediatrics. 2008;121(4):697-702.

151. Copenhagen TU. TSA - Trial Sequential Analysis. ctudk/tsa/2011.

\section{Publisher's Note}

Springer Nature remains neutral with regard to jurisdictional claims in published maps and institutional affiliations.

Ready to submit your research? Choose BMC and benefit from:

- fast, convenient online submission

- thorough peer review by experienced researchers in your field

- rapid publication on acceptance

- support for research data, including large and complex data types

- gold Open Access which fosters wider collaboration and increased citations

- maximum visibility for your research: over $100 \mathrm{M}$ website views per year

At BMC, research is always in progress.

Learn more biomedcentral.com/submissions 